\title{
STEROID CONSTITUENTS FROM LOBOPHYTUM CRASSUM
}

\author{
Nguyen Phuong Thao ${ }^{1}$, Nguyen Van Thanh ${ }^{1}$, Nguyen Hoai Nam ${ }^{1}$, Nguyen Xuan Cuong, \\ Young Ho Kim ${ }^{2}$, Phan Van Kiem ${ }^{1}$, Chau Van Minh ${ }^{1 *}$ \\ ${ }^{1}$ Institute of Marine Biochemistry, Vietnam Academy of Science and Technology \\ ${ }^{2}$ College of Pharmacy, Chungnam National University, Korea
}

Received 23 January 2015; Accepted for Publication 18 March 2015

\begin{abstract}
Using various chromatographic methods, three sterols, $(22 R, 23 R, 24 R)-5 \alpha, 8 \alpha$-epidioxy-22,23-methylene-24methylcholest-6-ene-3 $\beta$-ol (1), ergosterol peroxide (2), and 3 $\beta$-hydroxyandrost-5-ene-17-one (3), were isolated from the methanol extract of the soft coral Lobophytum crassum. Their structures were elucidated by 1D and 2D-NMR experiments and comparison of their NMR data with reported values. Compounds 1-3 were isolated from L. crassum for the first time.
\end{abstract}

Keywords. Lobophytum crassum, Alcyoniidae, soft coral, sterol.

\section{INTRODUCTION}

Among marine organisms, soft corals are known to elaborate both $3 \beta$-monohydroxysterols and polyhydroxysterols, derived mainly from a 24methylcholestane skeleton. Polyhydroxysterols of soft corals and other marine invertebrates occur mainly in either the free state or as the sulfate form, and examples of steroidal glycosides are rather rare, except for those found in starfishes [1]. As part of our continuing investigations to find bioactive compounds from Vietnamese marine organisms, this paper deals with the isolation from the soft coral Lobophytum crassum, and structure identification of three sterols: $(22 R, 23 R, 24 R)-5 \alpha, 8 \alpha$-epidioxy-22,23methylene-24-methylcholest-6-en-3 $\beta$-ol

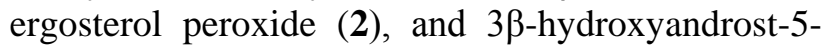
ene-17-one (3).

\section{EXPERIMENTAL}

\subsection{General experimental procedures}

The ${ }^{1} \mathrm{H}-\mathrm{NMR}(500 \mathrm{MHz})$ and ${ }^{13} \mathrm{C}-\mathrm{NMR}(125$ $\mathrm{MHz}$ ) spectra were recorded on a Bruker AM500 FT-NMR spectrometer, TMS was used as an internal standard. The electrospray ionization mass spectra (ESI-MS) were obtained on an Agilent 1260 series single quadrupole LC/MS system. Column chromatography (CC) was performed on silica gel (Kieselgel 60, 70-230 mesh and 230-400 mesh, Merck) and YMC RP-18 resins (30-50 $\mu \mathrm{m}$, Fuji
Silysia Chemical Ltd.). Thin layer chromatography (TLC) used pre-coated silica gel $60 \quad F_{254}$ (1.05554.0001, Merck) and RP-18 $\mathrm{F}_{254 \mathrm{~S}}$ plates (1.15685.0001, Merck). Compounds were visualized by spraying with aqueous $10 \% \mathrm{H}_{2} \mathrm{SO}_{4}$ and heating for 3-5 minutes.

\subsection{Marine materials}

The specimens of Lobophytum crassum were collected in Conco, Quang Tri, Vietnam, during May 2013 and deep frozen until used. The sample was identified by Professor Do Cong Thung (Institute of Marine Environment and Resources). A voucher of specimen (No. LC0513) was deposited at the Institute of Marine Biochemistry and the Institute of Marine Resources and Environment, VAST, Vietnam.

\subsection{Isolation}

Freeze-dried bodies of the soft coral $L$. crassum $(1.0 \mathrm{~kg})$ were well grinded and extracted three times with hot $\mathrm{MeOH}$ (at $50{ }^{\circ} \mathrm{C}$ for $5 \mathrm{~h}$ each time). The obtained solutions were filtered, combined, and concentrated under reduced pressure to yield a dark brown viscous residue $(75.0 \mathrm{~g}, \mathrm{~A})$. This residue was suspended in water $(1 \mathrm{~L})$ and partitioned in turn with $n$-hexane and $\mathrm{CH}_{2} \mathrm{Cl}_{2}(3 \times 1 \quad \mathrm{~L})$. The combined $\mathrm{CH}_{2} \mathrm{Cl}_{2}$ soluble portions were evaporated under reduced pressure to afford $\mathrm{CH}_{2} \mathrm{Cl}_{2}$ extract $(6.02 \mathrm{~g}$, B). The $\mathrm{CH}_{2} \mathrm{Cl}_{2}$ extract $(\mathrm{C}, 6.02 \mathrm{~g})$ was separated 
into five fractions, C1-C5, by a silica gel column chromatography (CC) using gradient elution of $n$ hexane-acetone (100:1-1:1, v/v). Fraction C3 (2.1 g) was further separated by $\mathrm{YMC} \mathrm{CC}$ using methanol-acetone-water $(2.5: 1: 1.1, \mathrm{v} / \mathrm{v} / \mathrm{v})$ to obtain six smaller fractions, C3A-C3F. Compounds 1 (9.5 $\mathrm{mg})$ and $2(8.2 \mathrm{mg})$ were purified from fraction C3D $(0.55 \mathrm{~g})$ by YMC RP-18 CC using methanolacetone-water $(3: 1: 1, \mathrm{v} / \mathrm{v} / \mathrm{v})$ as an eluent. Fraction C3C (0.39 g) was further separated by silica gel CC, eluted with chloroform-ethyl acetate $(30: 1, \mathrm{v} / \mathrm{v})$ to give compound $\mathbf{3}(11.3 \mathrm{mg})$.

$(22 R, 23 R, 24 R)-5 \alpha, 8 \alpha$-Epidioxy-22,23-

methylene-24-methylcholest-6-ene-3 $\beta$-ol (1): White powder; $[\alpha]_{\mathrm{D}}+30\left(c 0.1, \mathrm{CHCl}_{3}\right) ;{ }^{1} \mathrm{H}-\mathrm{NMR}(500$ $\left.\mathrm{MHz}, \mathrm{CDCl}_{3}\right)$ and ${ }^{13} \mathrm{C}-\mathrm{NMR}\left(125 \mathrm{MHz}, \mathrm{CDCl}_{3}\right)$ see Table 1; ESI-MS $m / z 443[\mathrm{M}+\mathrm{H}]^{+}\left(\mathrm{C}_{29} \mathrm{H}_{46} \mathrm{O}_{3}, \mathrm{M}=\right.$ 442).

Ergosterol peroxide (2): White powder; $[\alpha]_{\mathrm{D}}-30$ (c $\left.0.1, \mathrm{CHCl}_{3}\right) ;{ }^{1} \mathrm{H}-\mathrm{NMR}\left(500 \mathrm{MHz}, \mathrm{CDCl}_{3}\right.$ ) and ${ }^{13} \mathrm{C}-\mathrm{NMR}\left(125 \mathrm{MHz}, \mathrm{CDCl}_{3}\right)$ see table 1; ESI-MS $m / z 429[\mathrm{M}+\mathrm{H}]^{+}\left(\mathrm{C}_{28} \mathrm{H}_{44} \mathrm{O}_{3}, \mathrm{M}=428\right)$.

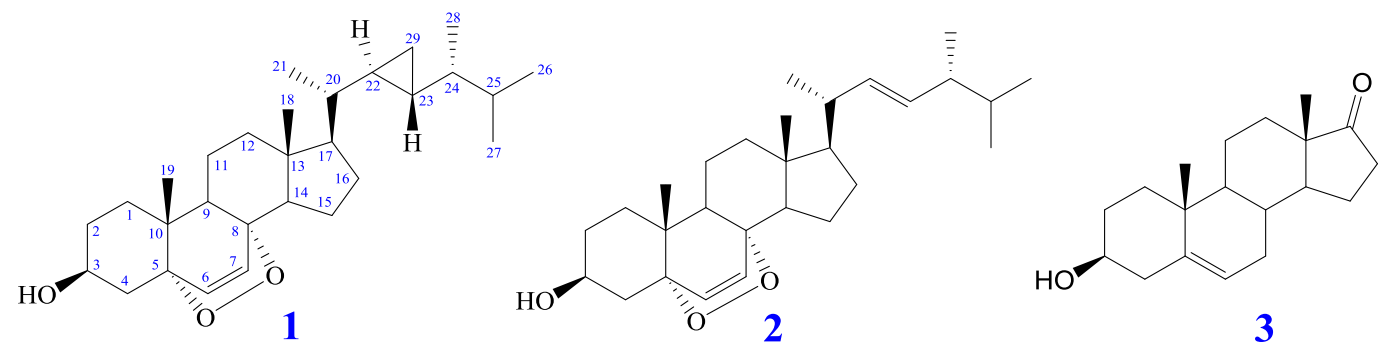

Figure 1: Chemical structures of compounds 1-3

Table 1: ${ }^{1} \mathrm{H}-\mathrm{NMR}(500 \mathrm{MHz})$ and ${ }^{13} \mathrm{C}-\mathrm{NMR}(125 \mathrm{MHz})$ data of $\mathbf{1}, \mathbf{2}$, and reported compounds

\begin{tabular}{|c|c|c|c|c|c|c|}
\hline \multirow{2}{*}{ C } & \multirow{2}{*}{${ }^{\mathrm{a}} \boldsymbol{\delta}_{\mathbf{C}}$} & \multicolumn{2}{|r|}{$1^{\mathrm{b}}$} & \multirow{2}{*}{${ }^{c} \boldsymbol{\delta}_{\mathrm{C}}$} & \multicolumn{2}{|r|}{$2^{b}$} \\
\hline & & $\boldsymbol{\delta}_{\mathrm{C}}$ & $\boldsymbol{\delta}_{\mathbf{H}}$ mult. $(J=\mathrm{Hz})$ & & $\boldsymbol{\delta}_{\mathrm{C}}$ & $\boldsymbol{\delta}_{\mathbf{H}}$ mult. $(J=\mathrm{Hz})$ \\
\hline 1 & 39.3 & 34.70 & $1.68 \mathrm{~m} / 1.94 \mathrm{~m}$ & 30.20 & 34.74 & $1.68 \mathrm{~m} / 1.96 \mathrm{~m}$ \\
\hline 2 & 30.1 & 30.09 & $1.53 \mathrm{~m} / 1.82 \mathrm{~m}$ & 34.77 & 30.17 & $1.55 \mathrm{~m} / 1.85 \mathrm{~m}$ \\
\hline 3 & 66.5 & 66.38 & $3.95 \mathrm{~m}$ & 66.47 & 66.50 & $3.97 \mathrm{~m}$ \\
\hline 4 & 51.1 & 36.94 & $1.90 \mathrm{~m} / 2.10 \mathrm{~m}$ & 39.44 & 37.01 & $1.92 \mathrm{~m} / 2.12 \mathrm{~m}$ \\
\hline 5 & 79.5 & 82.14 & - & 82.15 & 82.16 & - \\
\hline 6 & 130.8 & 135.39 & $6.22 \mathrm{~d}(8.5)$ & 135.22 & 135.44 & $6.24 \mathrm{~d}(8.5)$ \\
\hline 7 & 135.4 & 130.75 & $6.49 \mathrm{~d}(8.5)$ & 130.77 & 130.78 & $6.51 \mathrm{~d}(8.5)$ \\
\hline 8 & 82.2 & 79.48 & - & 79.42 & 79.44 & - \\
\hline 9 & 34.7 & 51.10 & $1.49 \mathrm{~m}$ & 51.25 & 51.17 & $1.49 \mathrm{~m}$ \\
\hline 10 & 36.9 & 36.92 & - & 37.03 & 36.99 & - \\
\hline 11 & 20.8 & 20.85 & $1.42 \mathrm{~m} / 1.63 \mathrm{~m}$ & 20.66 & 20.66 & $1.40 \mathrm{~m} / 1.60 \mathrm{~m}$ \\
\hline 12 & 39.6 & 39.34 & $1.22 \mathrm{~m} / 1.96 \mathrm{~m}$ & 37.03 & 39.40 & $1.23 \mathrm{~m} / 1.96 \mathrm{~m}$ \\
\hline 13 & 44.8 & 44.85 & - & 44.61 & 44.60 & - \\
\hline 14 & 51.3 & 51.34 & $1.54 \mathrm{~m}$ & 51.76 & 51.73 & $1.57 \mathrm{~m}$ \\
\hline 15 & 28.4 & 23.40 & $1.20 \mathrm{~m} / 1.50 \mathrm{~m}$ & 23.44 & 23.43 & $1.22 \mathrm{~m} / 1.52 \mathrm{~m}$ \\
\hline 16 & 23.4 & 28.47 & $1.50 \mathrm{~m} / 2.13 \mathrm{~m}$ & 28.58 & 28.64 & $1.36 \mathrm{~m} / 1.75 \mathrm{~m}$ \\
\hline 17 & 57.8 & 57.77 & $1.32 \mathrm{~m}$ & 56.33 & 56.27 & $1.23 \mathrm{~m}$ \\
\hline 18 & 12.4 & 12.46 & $0.75 \mathrm{~s}$ & 12.90 & 12.90 & $0.82 \mathrm{~s}$ \\
\hline 19 & 18.5 & 18.50 & $0.87 \mathrm{~s}$ & 18.18 & 18.19 & $0.88 \mathrm{~s}$ \\
\hline 20 & 39.5 & 39.56 & $0.82 \mathrm{~m}$ & 39.64 & 39.71 & $2.03 \mathrm{~m}$ \\
\hline 21 & 19.1 & 19.12 & 0.89 br s & 20.90 & 20.90 & $1.00 \mathrm{~d}(6.5)$ \\
\hline 22 & 24.2 & 24.16 & $0.53 \mathrm{~m}$ & 135.45 & 135.23 & $5.14 \mathrm{dd}(8.5,15.0)$ \\
\hline 23 & 25.1 & 25.12 & $0.30 \mathrm{~m}$ & 132.39 & 132.36 & $5.22 \mathrm{dd}(7.5,15.0)$ \\
\hline 24 & 45.0 & 45.00 & $0.52 \mathrm{~m}$ & 42.82 & 42.81 & $1.85 \mathrm{~m}$ \\
\hline 25 & 32.8 & 32.77 & $1.65 \mathrm{~m}$ & 33.11 & 33.10 & $1.47 \mathrm{~m}$ \\
\hline 26 & 18.1 & 18.14 & $0.85 \mathrm{~d}(7.0)$ & 19.64 & 19.65 & $0.82 \mathrm{~d}(6.5)$ \\
\hline 27 & 20.6 & 20.65 & $0.86 \mathrm{~d}(7.0)$ & 19.93 & 19.95 & $0.83 \mathrm{~d}(6.5)$ \\
\hline 28 & 15.7 & 15.74 & $0.90 \mathrm{~d}(7.0)$ & 17.56 & 17.57 & $0.90 \mathrm{~d}(6.5)$ \\
\hline 29 & 10.5 & 10.49 & $0.12 \mathrm{~m}$ & & & \\
\hline
\end{tabular}

${ }^{a} \delta_{\mathrm{C}}$ of $(22 R, 23 R, 24 R)-5 \alpha, 8 \alpha$-epidioxy-22,23-methylene-24-methylcholest-6-ene-3 $\beta$-ol [2], ${ }^{\mathrm{b}}$ recorded in $\mathrm{CDCl}_{3}$, ${ }^{\mathrm{c}} \delta_{\mathrm{C}}$ of ergosterol peroxide [3]. 
Table 2: NMR data of $\mathbf{3}$ and reported compound

\begin{tabular}{cccll}
\hline $\mathrm{C}$ & ${ }^{\mathrm{a}} \delta_{\mathrm{C}}$ & $\delta_{\mathrm{C}}{ }^{\mathrm{b}, \mathrm{c}}$ & $\delta_{\mathrm{C}}^{\mathrm{b}, \mathrm{d}}$ mult. $(J=\mathrm{Hz})$ & HMBC $(\mathrm{H} \rightarrow \mathrm{C})$ \\
\hline 1 & 37.8 & 37.20 & $1.10 \mathrm{~m} / 1.86 \mathrm{~m}$ & \\
2 & 32.1 & 31.59 & $1.52 \mathrm{~m} / 1.84 \mathrm{~m}$ & \\
3 & 71.3 & 71.59 & $3.53 \mathrm{~m}$ & $2,3,5,6,10$ \\
4 & 42.9 & 42.22 & $2.25 \mathrm{~m} / 2.33 \mathrm{~m}$ & \\
5 & 142.4 & 141.05 & - & \\
6 & 120.7 & 120.91 & $5.38 \mathrm{~m}$ & \\
7 & 32.1 & 30.79 & $1.64 \mathrm{~m} / 2.12 \mathrm{~m}$ & \\
8 & 32.1 & 31.52 & $1.68 \mathrm{~m}$ & \\
9 & 51.1 & 50.27 & $1.01 \mathrm{~m}$ & 13 \\
10 & 37.2 & 36.65 & - & $14,15,17$ \\
11 & 20.9 & 20.37 & $1.50 \mathrm{~m} / 1.68 \mathrm{~m}$ & \\
12 & 31.2 & 31.46 & $1.28 \mathrm{~m} / 1.84 \mathrm{~m}$ & \\
13 & 47.5 & 47.54 & - & $12,13,14,17$ \\
14 & 52.2 & 51.79 & $1.28 \mathrm{~m}$ & $1,5,9,10$ \\
15 & 22.2 & 21.88 & $1.55 / 1.95$ & \\
16 & 35.7 & 35.83 & $2.07 \mathrm{~m} / 2.46 \mathrm{~m}$ & \\
17 & 219.0 & 221.08 & - & \\
18 & 13.5 & 13.54 & $0.89 \mathrm{~s}$ & \\
19 & 19.5 & 19.42 & $1.04 \mathrm{~s}$ & \\
\hline
\end{tabular}

${ }^{\mathrm{a}} \delta_{\mathrm{C}}$ of 3ß-hydroxyandrost-5-ene-17-one [4], ${ }^{\mathrm{b}}$ recorded in $\mathrm{CDCl}_{3},{ }^{\mathrm{c}} 125 \mathrm{MHz},{ }^{\mathrm{d}} 500 \mathrm{MHz}$.

$3 \beta$-Hydroxyandrost-5-ene-17-one (3): White needles; mp. $140{ }^{\circ} \mathrm{C} ;[\alpha]_{\mathrm{D}}-5\left(c 0.1, \mathrm{CHCl}_{3}\right) ;{ }^{1} \mathrm{H}-$ NMR $\left(500 \mathrm{MHz}, \mathrm{CDCl}_{3}\right)$ and ${ }^{13} \mathrm{C}-\mathrm{NMR}(125 \mathrm{MHz}$, $\mathrm{CDCl}_{3}$ ) see table 2; ESI-MS $\mathrm{m} / \mathrm{z} 289[\mathrm{M}+\mathrm{H}]^{+}$ $\left(\mathrm{C}_{19} \mathrm{H}_{28} \mathrm{O}_{2}, \mathrm{M}=288\right)$.

\section{RESULTS AND DISCUSSION}

Compound 1 was obtained as a white powder. Its NMR features indicated a steroid, one main constituent of soft corals. The ${ }^{1} \mathrm{H}-\mathrm{NMR}$ spectrum showed singlets at $\delta_{\mathrm{H}} 0.75(\mathrm{H}-18)$ and $0.87(\mathrm{H}-19)$ assignable to two tertiary methyl groups, and doublets $(J=7.0 \mathrm{~Hz})$ at $\delta_{\mathrm{H}} 0.85(\mathrm{H}-26), 0.86(\mathrm{H}-27)$, and $0.90(\mathrm{H}-28)$ due to three secondary methyl groups. A sixth methyl signal appeared as a broad singlet at $\delta_{\mathrm{H}} 0.89$ and five high-field protons at $\delta_{\mathrm{H}}$ $0.53(1 \mathrm{H}, \mathrm{m}, \mathrm{H}-22), 0.30(1 \mathrm{H}, \mathrm{m}, \mathrm{H}-23), 0.52(1 \mathrm{H}$, $\mathrm{m}, \mathrm{H}-24)$, and $0.12(2 \mathrm{H}, \mathrm{m}, \mathrm{H}-29)$, suggesting for an ergosterol-type side chain possessing a cyclopropane ring [5]. In addition, 1 was recognized as a $5 \alpha, 8 \alpha$ epidioxy sterol by the presence of the characteristic doublet $(J=8.5 \mathrm{~Hz})$ proton signals for $\mathrm{H}-6$ at $\delta_{\mathrm{H}}$ 6.22 and $\mathrm{H}-7$ at $\delta_{\mathrm{H}} 6.49$ [6]. The ${ }^{13} \mathrm{C}-\mathrm{NMR}$ data of 1 (Table 1) were nearly identical to those of $(22 R, 23 R, 24 R)-5 \alpha, 8 \alpha$-epidioxy-22,23-methylene-24methylcholest-6-ene-3 $\beta$-ol [2]. However, based on HSQC and HMBC experiments (figure 2), the reported ${ }^{13} \mathrm{C}-\mathrm{NMR}$ data at C-1, C-4, C-5, C-6, C-7,
C-8, and C-9 [2] must be reassigned as shown in the table 1. Moreover, comparison of the ${ }^{13} \mathrm{C}-\mathrm{NMR}$ chemical shifts at $\mathrm{C}-15$ and $\mathrm{C}-16$ of $\mathbf{1}$ with those reported for similar compounds $[3,6,7]$ indicated that the published ${ }^{13} \mathrm{C}-\mathrm{NMR}$ data at $\mathrm{C}-15$ and $\mathrm{C}-16$ of $(22 R, 23 R, 24 R)-5 \alpha, 8 \alpha$-epidioxy-22,23-methylene24-methylcholest-6-ene-3 $\beta$-ol [2] must be reversed as shown in the table 1 .

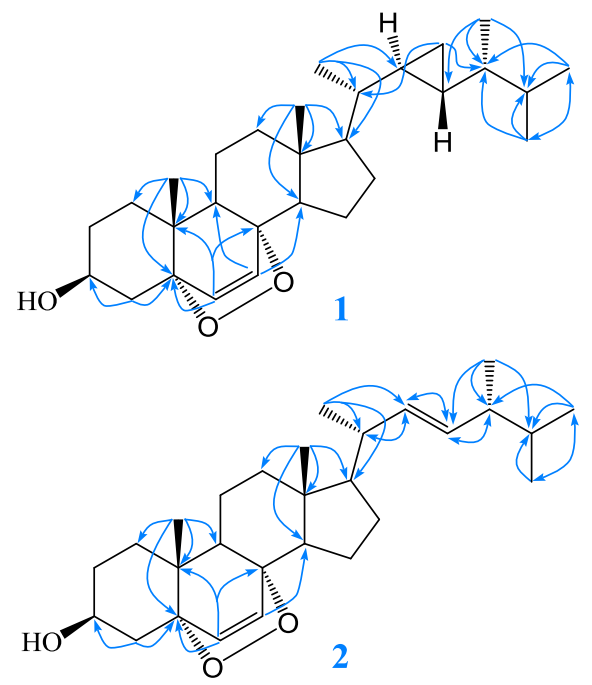

Figure 2: Key HMBC correlations of $\mathbf{1}$ and $\mathbf{2}$

The NMR spectroscopic data of $\mathbf{2}$ were similar to those of $\mathbf{1}$ and also indicative of a $5 \alpha, 8 \alpha$-epidioxy sterol. The difference of NMR data between these 
two compounds was only observed in the signals of the side chain with the presence of a disubstituted double bond at $\delta_{\mathrm{H}} 135.23(\mathrm{~d}, \mathrm{C}-22)$ and $132.36(\mathrm{~d}$, $\mathrm{C}-23) / \delta_{\mathrm{H}} 5.14(1 \mathrm{H}, \mathrm{dd}, J=8.5,15.0 \mathrm{~Hz}, \mathrm{H}-22)$ and $5.22(1 \mathrm{H}, \mathrm{dd}, J=7.5,15.0 \mathrm{~Hz}, \mathrm{H}-23)$ in 2 instead of a cyclopropane ring in $\mathbf{1}$. The large coupling constant $(J=15.0 \mathrm{~Hz})$ of the olefinic protons indicated a trans geometry of the double bond. From the above evidence, the ${ }^{13} \mathrm{C}$-NMR data of $\mathbf{2}$ were compared to those of ergosterol peroxide [3] and found to match (table 1). In addition, detailed analysis of HSQC and HMBC experiments led to determine compound $\mathbf{2}$ as ergosterol peroxide. As in case of compound 1, based on HSQC and HMBC experiments (Figure 2), the reported ${ }^{13} \mathrm{C}-\mathrm{NMR}$ data at $\mathrm{C} 1, \mathrm{C}-2, \mathrm{C}-4$, and $\mathrm{C}-12$ of 2 [3] must be reassigned as shown in the table 1 .

Compound $\mathbf{3}$ was elucidated as $3 \beta$ hydroxyandrost-5-ene-17-one [4] by an agreement of its ${ }^{13} \mathrm{C}$-NMR data with the reported values and combination with 2D-NMR data (table 2).

\section{CONCLUSION}

Using combined chromatographic methods, three sterols including $(22 R, 23 R, 24 R)-5 \alpha, 8 \alpha$ epidioxy-22,23-methylene-24-methylcholest-6-ene$3 \beta$-ol (1), ergosterol peroxide (2), and $3 \beta$ hydroxyandrost-5-ene-17-one (3) were isolated from the methanol extract of the soft coral Lobophytum crassum. Their structures were elucidated by spectroscopic methods. This is the first report of compounds 1-3 from L. crassum.

Acknowledgement. This research is funded by Vietnam National Foundation for Science and
Technology Development (NAFOSTED) under grant number 104.01-2012.37.

\section{REFERENCES}

1. M. Kobayashi, F. Kanda, S. R. Damarla, D. V. Rao, C. B. Rao. Marine sterols. XVII, Polyhdroxysterols of the soft corals of the Andaman and nicobar coasts. (2). Isolation and structures of three 16 $\beta$-hydroxy steroidal glycosides from an Alcyonium sp. soft coral, Chem. Pharm. Bull., 38(9), 2400-2403 (1990).

2. J. H. Sheu, K. C. Chang, C. Y. Duh. A cytotoxic 5a,8a-epidioxysterol from a soft coral Sinularia species. J. Nat. Prod., 63(1), 149-151 (2000).

3. J.-M. Yue, S.-N. Chen, Z.-W. Lin, H.-D. Sun. Sterols from the fungus Lactarium volemus, Phytochemistry, 56(8), 801-806 (2001).

4. J. W. Blunt, J. B. Stothers. ${ }^{13} C-N M R$ spectra of steroids - a survey and commentary. Org. Mag. Reson., 9(8), 439-464 (1977).

5. H. T. D'Armas, B. S. Mootoo, W. F. Reynolds. Steroidal compounds from the Caribbean octocoral Eunicea laciniata, J. Nat. Prod., 63(12), 1669-1671 (2000).

6. E. Ioannou, A. F. Abdel-Razik, M. Zervou, D. Christofidis, X. Alexi, C. Vagias, M. N. Alexis, V.

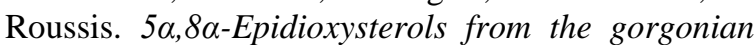
Eunicella cavolini and the ascidian Trididemnum inarmatum: Isolation and evaluation of their antiproliferative activity, Steroids, 74(1), 73-80 (2009).

7. X. Luo, F. Li, P. B. Shinde, J. Hong, C. O. Lee, K. S. Im, J. H. Jung. 26,27-Cyclosterols and other polyoxygenated sterols from a marine sponge Topsentia sp., J. Nat. Prod., 69(12), 1760-1768 (2006).

\section{Corresponding author: Chau Van Minh}

Institute of Marine Biochemistry, Vietnam Academy of Science and Technology

18 Hoang Quoc Viet, Cau Giay, Hanoi, Vietnam

E-mail: cvminh@vast.vn. 